\title{
C3 GLOMERULONEPHRITIS AND DIGITAL ISCHEMIA AS THE INITIAL CLINICAL MANIFESTATION OF MONOCLONAL GAMMOPATHY: CASE REPORT
}

Pablo Vinicius da Fonseca ${ }^{1, *}$, Jean Michell Correia Monteiro ${ }^{1}$, Victor Matheus Ostrovski Souza Santos ${ }^{1}$, Henrique Ayres Mayrink Giardini $^{1}$, Lissiane Karine Noronha Guedes ${ }^{1}$, Rosa Maria Rodrigues Pereira ${ }^{1}$

1. Universidade de São Paulo, São Paulo (SP), Brazil.

*Corresponding author: pabloviniciusfonseca@gmail.com

\section{BACKGROUND}

C3 glomerulopathy is a rare entity that manifests as membranoproliferative glomerulonephritis and, in immunohistochemistry, C3 glomerular deposit. Monoclonal gammopathy represents one of the causes, probably because it interferes with the regulation of the alternative complement pathway. Digital ischemia due to thrombosing vasculopathy without evidence of hyperviscosity, cryoglobulinemic vasculitis or cryofibrinogenemia is not a common manifestation in patients with monoclonal gammopathy.

\section{CASE REPORT}

Female patient, dark-skinned, 25 years old, previously healthy, started in March 2016 with cyanosis and pain in the fingers of the lower limbs after exposure to cold, evolving with subsequent necrosis and tissue loss. Initial laboratory evaluation showed mild renal failure (creatinine $1.5 \mathrm{mg} / \mathrm{dL}$ ), dysmorphic hematuria and subnephrotic proteinuria (0.34 g/24 h), anemia ( $\mathrm{Hb} 9.9 \mathrm{~g} / \mathrm{dL}$, secondary to hemoglobinopathy C), and IgG/Kappa monoclonal gammopathy (monoclonal component $0.4 \mathrm{~g} / \mathrm{dL}$, only serum, considered low risk).

Due to the suspicion of systemic vasculitis, pulse therapy with methylprednisolone was performed followed by intravenous human immunoglobulin (IVIG), cyclophosphamide and, afterwards, rituximab. She remained temporarily stable, with no new ischemic events, but maintained dysmorphic hematuria with baseline creatinine of $1.4 \mathrm{mg} / \mathrm{dL}$.

Six months after the patient developed new digital ischemias and persistent hematuria and proteinuria. Renal biopsy was performed which showed proliferative glomerulonephritis with only $\mathrm{C} 3$ deposition in immunofluorescence, and skin biopsy of the affected finger which showed thrombosing vasculopathy. She received IVIG, prednisone, azathioprine and rituximab.

In April 2020, days after the diagnosis of COVID-19, she developed new digital ischemias and worsening renal function (creatinine $3.3 \mathrm{mg} / \mathrm{dL}$, proteinuria $0.85 \mathrm{~g} / 24 \mathrm{~h}$, dysmorphic hematuria). Hypothesis of thrombosing digital vasculopathy and C3 glomerulonephritis secondary to monoclonal gammopathy, with negative cryoglobulins and cryofibrinogen research. Anticoagulation with enoxaparin and five plasmapheresis sessions were initiated, with stabilization of digital vasculopathy. Marrow evaluation showed $0.53 \%$ of monoclonal plasma cells and tomography of the chest, abdomen and pelvis without signs of lymphoproliferation.

Due to the absence of criteria for hematological cancer, a new attempt at immunosuppression was performed with prednisone, rituximab and mycophenolate mofetil. 


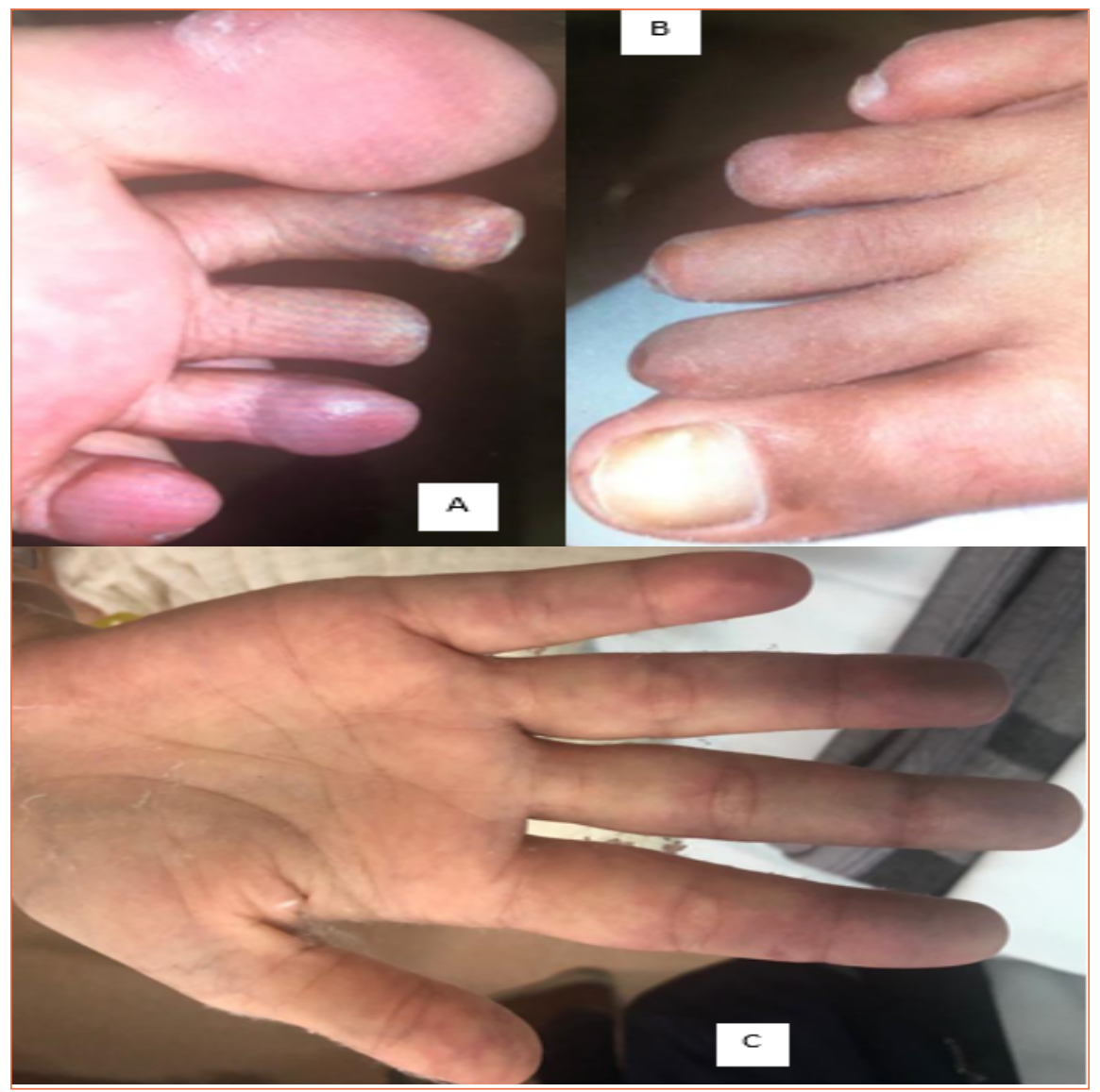

Figure 1. (A) Left foot, cyanosis and distal phalange amputation of the 3rd toe; (B) Right foot, cyanosis and amputation of the distal phalanges of the 2nd, 3rd and 4th toes; (C) Right hand, cyanosis - most important in 2nd, 3rd and 4th fingers.

\section{CONCLUSION}

C3 glomerulopathy is a rare condition, related to inadequate activation of the complement system with renal deposition of C3 and consequent membranoproliferative glomerulonephritis. The ideal treatment is not yet defined, with variable results in relation to immunosuppression and chemotherapy directed to the plasmacytic clone. The presence of thrombosing vasculopathy in patients with monoclonal gammopathy should always lead to the investigation of hyperviscosity, cryoglobulinemia and cryofibrinogenemia. 